\title{
LA ESTRUCTURA Y FUNCIÓN DE LAS COLINESTERASAS: BLANCO DE LOS PLAGUICIDAS
}

\author{
Rosa María LÓPEZ-DURÁN ${ }^{1,3}$, Rafael VALENCIA-QUINTANA ${ }^{2,3}$, Juana SÁNCHEZ-ALARCÓN²,3, \\ Benjamín PÉREZ-AGUILAR ${ }^{1}$,Noe SALINAS-ARREORTUA ${ }^{1}$, Héctor SERRANO ${ }^{1}$, \\ María Dolores GARCÍA-SUÁREZ ${ }^{4}$, Hipólito MUÑOZ-NAVA², Ángel HERNÁNDEZ-HERNÁNDEZ ${ }^{2}$, \\ Cecilio VIDAL-MORENO ${ }^{5}$ y José Luis GÓMEZ-OLIVARES ${ }^{1,3 *}$
}

${ }^{1}$ Departamento de Ciencias de la Salud, División de Ciencias Biológicas y de la Salud, Universidad Autónoma Metropolitana-Iztapalapa, México

${ }^{2}$ Facultad de Agrobiología, Universidad Autónoma de Tlaxcala, México

${ }^{3}$ Red Temática "La Toxicidad de los Plaguicidas", Consejo Nacional de Ciencia y Tecnología-Universidad Autónoma de Nayarit, México

${ }^{4}$ Departamento de Biología, División de Ciencias Biológicas y de la Salud, Universidad Autónoma Metropolitana-Iztapalapa, México

${ }^{5}$ Departamento de Bioquímica y Biología Molecular A, Campus Espinardo, Universidad de Murcia, España

*Autor para correspondencia: gool@xanum.uam.mx

(Recibido marzo 2017; aceptado junio 2018)

Palabras clave: acetilcolinesterasa, butirilcolinesterasa, paraoxonasa, plaguicidas

\section{RESUMEN}

En esta revisión sobre las colinesterasas se abarcan temas tales como las características de los genes y los transcritos generados durante el proceso de corte-empalme alternativo que deriva en una amplia heterogeneidad estructural proteica - polimorfismo molecular-; se hace una descripción detallada de la estructura proteínica implicada en el mecanismo de actividad enzimática; se mencionan evidencias que sustentan algunas funciones enzimáticas alternativas, y se examinan tanto el mecanismo de inhibición general por los plaguicidas organofosforados y carbamatos como sus efectos fisiológicos.

Key words: acetylcholinesterase, butyrylcholinesterase, paraoxonase, pesticides

\begin{abstract}
In this review on cholinesterases we cover topics such as the characteristics of genes and the transcripts generated during an alternative splicing process that results in a broad structural heterogeneity protein (molecular polymorphism); we offer a detailed description of the structure protein involved in the mechanism of enzymatic activity; we provide evidence supporting some alternative functions, and we analyze the mechanisms of inhibition by organophosphorus pesticides and carbamates, as well as their physiological effects.
\end{abstract}




\section{INTRODUCCIÓN}

El plasma humano contiene más de 10000 proteínas, las cuales se han identificado por espectrometría de masas (Lockridge 2015). Las esterasas en el plasma humano son butirilcolinesterasa, paraoxonasa $1 \mathrm{y}$, en menor cantidad, acetilcolinesterasa. Las esterasas se han clasificado como tipo A, aquellas que contienen un residuo de cisteína en el centro activo, y las de tipo B, que contienen un residuo de serina (Dvir et al. 2010).

En las esterasas tipo A, los organofosforados interaccionan con el grupo funcional -SH y forman un enlace P-S que es fácilmente hidrolizado, mientras que en las esterasas tipo B, la interacción es con el -OH del residuo de serina, formando un enlace P-O que no es hidrolizado (Dvir et al. 2010).

\section{PROPIEDADES GENERALES DE LAS COLINESTERASAS}

Las colinesterasas (ChEs) poseen una capacidad extraordinaria para hidrolizar ésteres de colina con mayor rapidez que a otros ésteres, cuando se comparan las velocidades de hidrólisis en condiciones óptimas de concentración de substrato, $\mathrm{pH}$ y fuerza iónica, usando preparaciones desprovistas de otras esterasas. Además, se diferencian claramente de otras esterasas por la inhibición que sufren con pequeñas cantidades $\left(10^{-5} \mathrm{M}\right)$ del alcaloide natural fisostigmina (eserina) (Triggle et al. 1998).

Los vertebrados presentan dos tipos de colinesterasas: acetilcolinesterasa o colinesterasa verdadera (AChE) y butirilcolinesterasa (BChE o BuChE), también llamada pseudocolinesterasa, colinesterasa plasmática o sérica (Massoulié y Toutant 1988).

La primera clasificación de las ChEs procede de los resultados obtenidos tras los análisis de enzimas de sangre completa y suero, con el propósito de determinar el sustrato preferente para cada una de ellas (Gahler y Plattner 1927). Stedman et al. (1932) obtuvieron de suero de caballo una preparación enzimática con alta especificidad para hidrolizar ésteres de colina, aunque con mayor capacidad para hidrolizar butirilcolina que acetilcolina. Estos resultados fueron corroborados por Glick (1941), quien observó que en el suero humano la actividad colinesterásica aumentaba conforme crecía la longitud de la cadena acilo del sustrato hasta cuatro carbonos, y disminuía con longitudes superiores.

\section{Actividad de acetilcolinesterasa}

Desde 1914, año en que fue descubierta su actividad, la AChE enzima ha estado en el interés de gran número de laboratorios en el mundo. La investigación sobre esta enzima se ha enfocado a conocer las diversas funciones biológicas catalíticas y no catalíticas en las que puede estar implicada, que incluyen la función clásica de la eliminación de la acetilcolina en las uniones nerviosa y neuromuscular. La AChE posee una amplia distribución y localización tisular (Dale 1914), y actualmente se ha establecido que todos los tejidos del cuerpo humano poseen la capacidad de sintetizarla (Layer y Willbold, 1995).

A partir de la síntesis de los compuestos anticolinesterásicos en el siglo XIX se ha observado que existe una relación entre la actividad colinesterásica y la farmacología. En sus estudios sobre los componentes del sistema colinérgico, Sir Henry Dale sugirió que la fisostigmina inhibía una enzima que catalizaba la hidrólisis de ésteres de colina (Dale 1914).

$\mathrm{La} \mathrm{AChE}$ es una serina-hidrolasa que escinde ésteres de colina, siendo su principal substrato el neurotransmisor acetilcolina (ACh). Posee una alta actividad catalítica, y cada molécula es capaz de degradar cerca de 25000 moléculas de ACh por segundo. Se le considera una enzima casi perfecta cuyo límite de trabajo depende de la difusión del sustrato (Quinn 1987, Taylor y Radic 1994). Diversos estudios de cinética enzimática señalan que en el sitio activo de la $\mathrm{AChE}$ existen regiones estructurales muy bien definidas. Se considera que dicho sitio está compuesto por dos subsitios: $a$ ) esterásico y $b$ ) aniónico (Nachmansohn y Wilson 1951, Sussman et al. 1991, 1993). Ambos son esenciales en el mecanismo catalítico desarrollado por la enzima. El subsitio esterásico contiene la triada catalítica compuesta por los residuos de Ser200, His400 y Glu327. Es en este último donde se hidroliza la ACh, generándose acetato y colina (Sussman et al. 1991).

La hidrólisis de la acetilcolina (ChO-COR) por la $\mathrm{AChE}(\mathrm{AChEOH})$ incluye dos etapas: en la primera se forma un intermediario acil-enzima (AChEOCOR), una vez que el grupo acilo queda unido de modo covalente a la serina del centro activo (Ser200). En la segunda etapa se libera el grupo acilo (Schwarz et al. 1995).

$$
\begin{array}{r}
\mathrm{Cho}-\mathrm{COR}+\mathrm{AChEOH} \rightarrow \mathrm{AChEO}-\mathrm{COR}+\mathrm{ChOH} \\
\mathrm{AChEO}-\mathrm{COR}+\mathrm{OH}^{-} \rightarrow-\mathrm{OCOR}+\mathrm{AChEOH}
\end{array}
$$

La AChE promueve la escisión de la ACh para formar acil-enzima y colina libre. La acil-colina sufre un ataque nucleofílico por una molécula de agua, 
asistida por un residuo de His440; como resultado libera acetato y ocurre la regeneración del sitio activo de la enzima (Soreq y Seidman 2001, Pohanka 2011).

El subsitio aniónico está definido por los residuos Trp84, Phe330 y Phe331, e interactúa con la estructura de la ACh a través del grupo amino cuaternario cargado positivamente (Dvir et al. 2010).

Los sustratos catiónicos no se unen por un residuo de aminoácido al sitio aniónico, sino con 14 residuos de aminoácidos aromáticos que se alinean con la acetilcolina para que quede próxima a la Ser del centro activo (Radic et al. 1992, Ordentlich et al. 1993, Sussman et al. 1993, Ariel et al. 1998). Algunos residuos de aminoácidos aromáticos son indispensables para la actividad enzimática, como en el caso del residuo triptófano 84 (Sussman et al. 1991, Ordentlich et al. 1998, Dvir et al. 2010).

Cabe señalar que además de los dos subsitios descritos en el centro catalítico, la AChE posee un sitio de unión adicional para la $\mathrm{ACh}$ y ligandos cuaternarios, el cual se denomina sitio aniónico periférico (Taylor y Lappi 1975).

\section{El gen de acetilcolinesterasa y el procesamiento transcripcional}

En humanos, el gen $A C H E$ está localizado en la región q22 del cromosoma 7. La longitud del gen es de $7 \mathrm{~kb}$ y está constituido por seis exones y cuatro intrones (Getman et al. 1992). A través de corte-empalme alternativo en la región 3' del gen se generan tres transcritos diferentes (Massoulié et al. 1998).

A este respecto, la isoforma tetramérica $\mathrm{AChE}-\mathrm{S}$ ("S" indica sináptica; esta isoforma también se conoce como AChE-T, donde "T" indica "con tallo"). La AChE-S es el producto de los exones 2, 3, 4 y 6 , presentando una región carboxilo terminal que contiene cisteínas (Massoulié et al. 1993).

Otra variante del transcrito, la AChE-E ("E" indica eritrocítica; también se conoce como AChE-H ["H" indica hidrofóbica]), está compuesta por los exones 2, 3, 4 y 5 (Coussen et al. 2001).

Finalmente, el proceso de corte-empalme alternativo en la región 3", genera el transcrito "readthrough" AChE (AChE-R) (Kaufer et al. 1998, Meshorer et al. 2002) que está compuesto por los exones 2, 3, 4, 5, 6 y el intrón 4 (Fig. 1) (Soreq y Seidman 2001, Massoulié 2002) y codifica un monómero.

\section{Heterogeneidad estructural de la acetilcolines- terasa}

La acetilcolinesterasa muestra una amplia heterogeneidad estructural. El transcrito AChE-S codifica a monómeros, dímeros y tetrámeros anfifílicos $\left(\mathrm{G}_{1}{ }^{\mathrm{A}}\right.$,
$\left.\mathrm{G}_{2}{ }^{\mathrm{A}} \mathrm{y} \mathrm{G}_{4}{ }^{\mathrm{A}}\right)$, tetrámeros hidrofílicos $\left(\mathrm{G}_{4}{ }^{\mathrm{H}}\right)$, y asociaciones hetero-oligoméricas (Bon et al. 1997). Si los tetrámeros están unidos a una proteína transmembranal que contiene residuos de prolina denominado PRiMA (Proline Rich Membrane Anchor), se conocen como tetrámeros anfifílicos, capaces de presentar inserción en las membranas (PRiMA-G ${ }_{4}{ }^{\mathrm{A}}$ ) (Perrier et al. 2002). Si los tetrámeros están unidos a un tallo proteico tipo colágeno (ColQ), son generadas las formas asimétricas $\mathrm{A}_{4}, \mathrm{~A}_{8}$ y $\mathrm{A}_{12}$, con uno, dos o tres tetrámeros, respectivamente (Fig. 1) (Massoulié 2002).

El transcrito E (eritrocítico) o H (hidrofóbico) genera dímeros anfifílicos tipo I, en los que cada subunidad está unida de manera covalente a un resto de glicofosfatidilinositol, el cual es incorporado en el retículo endoplásmico previo a la formación de los dímeros (Fig. 1) (Coussen et al. 2001). Éste tipo de forma molecular es característico de células hematopoyéticas (Gómez et al. 2003). Diversos tejidos entre los que se incluyen corazón, hígado, pulmón, riñón, intestino, timo y bazo, expresan dímeros y monómeros (Gómez et al. 1999, 2000, Ruiz-Espejo et al. 2002, Nieto-Cerón et al. 2005, Moral-Naranjo et al. 2010, Muñoz-Delgado et al. 2010, Montenegro et al. 2014).

El transcrito AChE-R codifica una proteína que tiene una región carboxilo terminal más corta y carece de residuos de cisteína; así, la isoforma AChE-R es un monómero hidrosoluble (Fig. 1). La proteína AChE-R se ha detectado en cerebro de mamífero y humano (Kaufer et al.1998, Soreq and Seidman 2001, Massoulié 2002, Meshorer et al. 2002).

Además del proceso de corte-empalme alternativo descrito en la figura 1, en el extremo 3' del gen de acetilcolinesterasa humano, se ha observado que ocurre una compleja regulación que está implicada en la diversidad de transcritos AChE (Fig. 1) (Meshorer et al. 2004).

\section{Papel biológico de la acetilcolinesterasa}

El principal papel de la $\mathrm{AChE}$ es la inactivación rápida y precisa del neurotransmisor acetilcolina después de su liberación hacia las sinapsis colinérgicas, finalizando los impulsos en la transmisión nerviosa y facilitando el control temporal preciso de la contracción muscular (Rosenberry 1979, Taylor 1991). Además, se han descrito diversas funciones alternativas de la $\mathrm{AChE}$ en algunos procesos celulares que se describen a continuación.

\section{Proceso de apoptosis}

Una función alternativa relevante de la AChE ocurre en el proceso de apoptosis. Se ha establecido que 
A

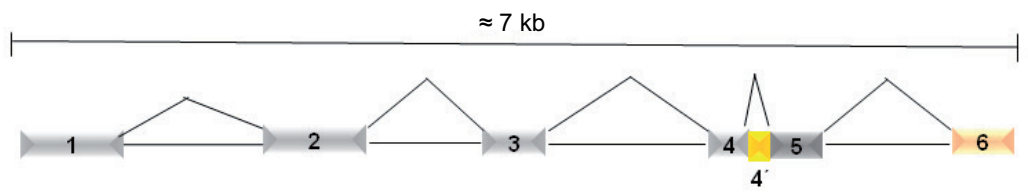

B

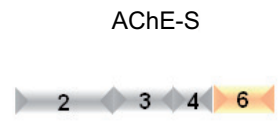

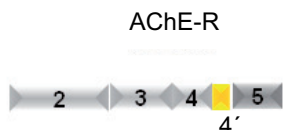

$4^{\prime}$

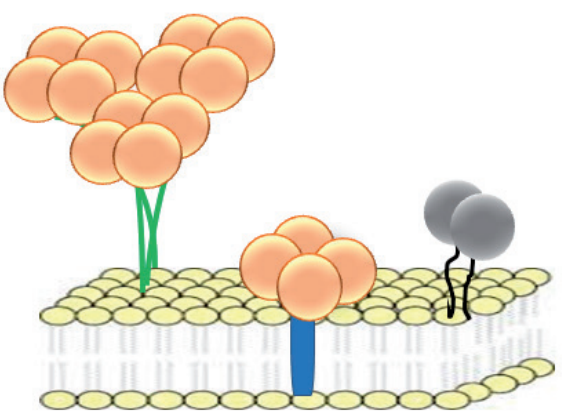

AChE-E
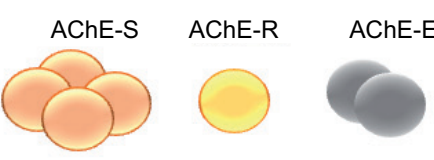

Fig. 1. Estructura del gen $A C H E$ humano, procesamiento de corte-empalme alternativo y productos proteicos. A) Proceso de corte-empalme de exones (rectángulos) e intrones (líneas horizontales). El 4' pseudo-intrón 4 se indica en color amarillo. B) Variantes de transcritos: AChE-S, AChE-E y AChE-R. C) Polimorfismo molecular de la proteína AChE. AChE-S: formas globulares monoméricas, diméricas y tetraméricas hidrosolubles (subunidad catalítica en color naranja); forma tetramérica anclada a PRiMA (péptido hidrofóbico en color azul) o tallo tipo colágeno (en líneas verde). AChE-E: forma molecular dimérica (negra) anclada a membrana por enlace glicofosfatidilinositol (hexágono color rojo). AChE-S, AChE-E, AChE-R: formas moleculares monoméricas ancladas a membrana por hélice alfa

su expresión puede inducirse durante la apoptosis en diferentes tipos celulares, incluyendo células que no se originan en el sistema nervioso. Los fibroblastos de pulmón humano y las células de riñón de rata no muestran actividad AChE, pero se incrementa significativamente su expresión cuando sufren apoptosis (Zhang et al. 2002, Jin et al. 2004). Se ha observado que células PC12 expresan baja actividad AChE, y cuando se induce apoptosis de manera experimental, hay aumento de esta actividad (Yang et al. 2002, Jin et al. 2004).

Se ha establecido una relación entre la presencia de la AChE y el proceso de apoptosis. Park et al. (2004) mostraron que el silenciamiento de la AChE provoca una inhibición en la interacción entre Apaf1 y el citocromo c, dos proteínas importantes en la apoptosis. Con sus experimentos demostraron que la AChE juega un papel importante en la formación del apoptosoma. Esto lo confirmaron cuando emplearon ARN interferente (siRNA, por sus siglas en inglés) contra el gen del citocromo $\mathrm{C}$ donde se suprimió la interacción entre AChE y Apaf-1, en tanto que el uso de siRNA contra el gen Apaf-1 no bloqueó la interacción entre AChE y el citocromo C (Park et al. 2004).

Posteriormente, Park et al. (2008) demostraron que la AChE interacciona con la caveolina-1, y subsecuentemente con el citocromo $\mathrm{C}$, que es indispensable para la formación del apoptosoma.

\section{Progresión del ciclo celular}

Recientemente, se ha asociado un aumento en el contenido de AChE con los mecanismos de regulación del ciclo celular. El proceso previo a la diferenciación es la proliferación, por lo que podría ser necesario inhibir la proliferación celular para entrar al proceso de diferenciación. Las evidencias indican que el contenido de $\mathrm{AChE}$ aumenta durante la diferenciación de tejidos no neuronales, tales como hueso, músculo y hematopoyético (Lev-Lehman et al. 1997, Genever et al. 1999, Serobyan et al. 2007). Se ha sugerido que tal aumento en la $\mathrm{AChE}$ es indispensable para que las células ingresen en el proceso de diferenciación. Por ejemplo, en el caso de células Caco-2, cuando se indujo una sobre-expresión de la AChE se observó incremento en el porcentaje de células en la fase $\mathrm{G}_{2} / \mathrm{M}$ del ciclo celular, mientras que las células restantes (34\%) se encontraban en otras fases del ciclo. Estos datos sugirieron que la AChE induce arresto en el ciclo celular en la fase $\mathrm{G}_{2} / \mathrm{M}$ (Xiang et al. 2008). Resultados similares se 
han encontrado en células R28 cuando se induce la sobre-expresión de AChE (Layer et al. 2013), lo cual sustenta su papel en la progresión del ciclo celular. Experimentos de co-inmunoprecipitación revelan que la AChE interactúa con la ciclina $\mathrm{G}$ asociada a cinasa y Aurora cinasa. Junto con un análisis de predicción de secuencia de interacción y/o modificaciones en proteínas, se encontró que el extremo N-terminal de la AChE posee un sitio de unión a ciclinas (Toiber et al. 2009), que puede explicar su participación en el arresto en alguna fase del ciclo celular, a través de una interacción directa con proteínas reguladoras de tal proceso celular.

En el mismo sentido, existen evidencias experimentales que implican una alteración de la actividad enzimática $\mathrm{AChE}$ con una promoción en la proliferación celular e inhibición del proceso de apoptosis, por la activación de los receptores muscarínicos y nicotínicos (Thunnissen 2009), la cual depende de los niveles de la acetilcolina. Por lo anterior, una disminución de la actividad AChE podría promover un aumento de acetilcolina a niveles locales.

A este respecto, Cassiman et al. (2002) demostraron en ratas que el nervio vago estimula la activación de células progenitoras hepáticas a través del receptor muscarínico tipo 3 al realizar una vagotomía, en la cual observaron disminución del contenido de AChE que facilitó la llegada de la acetilcolina a las células ovales para promover la proliferación celular y la reparación del hígado. Finalmente, se ha demostrado en las líneas celulares de cáncer de hígado Huh-7 y HepG2, que la inhibición de la actividad AChE causó un aumento en la tasa de proliferación de dichos tipos celulares (Pérez-Aguilar et al. 2015), lo cual sustenta el papel de la AChE en el control del ciclo celular.

Estudios epidemiológicos han mostrado que el uso ocupacional de plaguicidas que inhiben la actividad AChE aumenta el riesgo de leucemia (Vidal 2005), linfoma (Navaranjan et al. 2013) y otros tipos de cáncer (Alavanja et al. 2004). Las evidencias experimentales que sustentan esta relación proceden de proporcionar a ratas un tratamiento con plaguicidas organofosforados que inhiben la AChE, produciéndose carcinomas (Vidal 2005). En leucemia, cáncer de ovario (Soreq et al. 1991), cáncer de mama (Bernardi et al. 2010) y cáncer de pulmón (Vidal 2005) se han determinado amplificaciones y/o deleciones, así como alteraciones estructurales en los genes AChE y BuChE (Soreq et al. 1991).

\section{Actividad butirilcolinesterasa}

Desde la década de 1940 se ha estudiado la actividad BuChE (Alles y Hawes 1940), siendo aquél el momento en que se estableció la existencia de dos colinesterasas. En la década de 1950, con la introducción de la succinilcolina como relajante muscular, se encontró que algunos humanos presentaban una forma hereditaria de $\mathrm{BuChE}$ que provocaba incapacidad de hidrolizarla (Lehmann y Ryan 1956, Kalow y Staron 1957).

La BuChE cataliza la hidrólisis de esteres de colina, incluyendo butirilcolina, succinilcolina y acetilcolina. La BuChE está estructural y funcionalmente relacionada con la AChE (Massoulié et al. 1993, Taylor y Radic 1994). Ambas enzimas presentan un residuo de serina que es esencial para su actividad catalítica (Cohen y Oosterbaan 1963). Como se señaló, la BuChE hidroliza acetilcolina, pero lo hace con menor eficacia que la $\mathrm{AChE}$ ( $\mathrm{Li}$ et al. 2000, Mesulam et al. 2002).

Cada subunidad catalítica de BuChE humana está compuesta de 574 residuos de aminoácidos. Por estudios de mutagénesis dirigida se han identificado residuos de aminoácidos que están implicados en su capacidad catalítica y la unión de inhibidores de la enzima (Radic et al. 1993).

El sitio activo se encuentra ubicado al fondo de la garganta catalítica delineada por 55 residuos de aminoácidos. El sitio aniónico periférico está localizado en la boca de la garganta. Los residuos de aminoácidos Asp70 y Tyr332 del PAS están involucrados en la unión inicial de los sustratos cargados positivamente, como el amonio cuaternario que contienen los esteres de colina. La estructura funcional de garganta del sitio activo de la BuChE está controlada por un puente de hidrógeno formado entre Asp70 y Tyr322 (Çokuğraş 2003).

$\mathrm{La}$ actividad BuChE se engloba en la familia de las serina-hidrolasas, debido a que incluye un residuo de serina en el sitio activo. La catálisis por $\mathrm{BuChE}$ requiere una triada catalítica: Ser198, Glu325, e His438 (Nicolet et al. 2003).

$\mathrm{La}$ actividad $\mathrm{BuChE}$ es inhibida irreversiblemente por compuestos organofosforados, tales como el agente nervioso sarín y plaguicidas como el clorpirifos. La serina del sitio activo se modifica covalentemente al reaccionar con agentes organofosforados inactivando la BuChE (Jansz et al. 1959, Fidder et al. 2002).

\section{El gen de butirilcolinesterasa y el procesamiento transcripcional}

En el genoma humano, el gen $B C H E$ se encuentra en el cromosoma 3, en la posición q26.1-q26.2 (Allderdice et al. 1991, Gaughan et al. 1991). La región genómica del gen $B C H E$ tiene una longitud de 
$64.57 \mathrm{~kb}$ y está compuesto por 4 exones y 3 intrones (Arpagaus et al. 1990, Massoulie 2002).

Se han identificado más de 40 mutaciones en el gen $B C H E$, que generan enzimas con diferentes niveles de actividad catalítica (La Du et al. 1990). La enzima normal es sensible a dibucaína. Una mutación en el codón 70 del gen $B C H E$ causa la substitución de un residuo de ácido aspártico por una glicina (D70G). La enzima producida es una variante insensible en un $30 \%$ a la inhibición por dibucaína (McGuire et al. 1989).

\section{Heterogeneidad estructural de la $\mathrm{BuChE}$}

Todas las formas moleculares de $\mathrm{BuChE}$ derivan de un solo mRNA, que no sufre un empalme alternativo (Darvesh et al. 2003). La proteína codificada en el mRNA BuChE-T (con tallo) generado de la expresión del gen $B C h E$ refleja diferentes formas moleculares que están compuestas por subunidades catalíticas idénticas, entre las que se incluyen monómeros $\left(\mathrm{G}_{1}{ }^{\mathrm{H}}\right)$, dímeros $\left(\mathrm{G}_{2}{ }^{\mathrm{H}}\right)$ y tetrámeros $\left(\mathrm{G}_{4}{ }^{\mathrm{H}}\right)$ hidrofílicos; formas anfifílicas, y monómeros $\left(\mathrm{G}_{1}{ }^{\mathrm{A}}\right)$, dímeros
$\left(\mathrm{G}_{2}{ }^{\mathrm{A}}\right)$ y tetrámeros $\left(\mathrm{G}_{4}{ }^{\mathrm{A}}\right)$, estos últimos anclados a la membrana por un péptido hidrofóbico rico en prolina (PRiMA). Se presentan formas asimétricas ancladas a membrana por una triple hélice tipo colágeno, a la que están anclados uno, dos o tres tetrámeros, conocidos como $\mathrm{A}_{4}, \mathrm{~A}_{8} \mathrm{y} \mathrm{A}_{12}$, respectivamente (Darvesh et al. 2003). Esta amplia heterogeneidad estructural de formas moleculares en la proteína $\mathrm{BuChE}$ es muy similar a lo descrito para el transcito AChE-S (Fig. 2). En mamíferos, la proteína $\mathrm{BuChE}$ se presenta en plasma, hígado y corazón. Se considera que el hígado es el órgano productor de la proteína $\mathrm{BuChE}$ que se detecta en el plasma (Gómez et al. 1999, 2000).

\section{Papel biológico de la BuChE}

Por el hecho de que las personas con variantes "silenciosas" - incapacidad de catalizar la hidrólisis de esteres de colina - de BuChE no muestran alteraciones fisiológicas, se sugirió que la enzima no tenía un papel biológico establecido.

Como se ha señalado previamente, la $\mathrm{BuChE}$ puede catalizar la hidrólisis de acetilcolina. En el cerebro

A

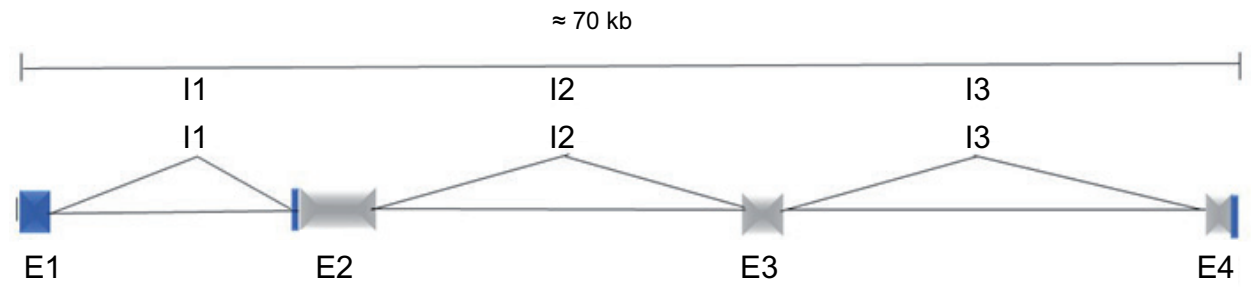

B

E2 E3 E4

C
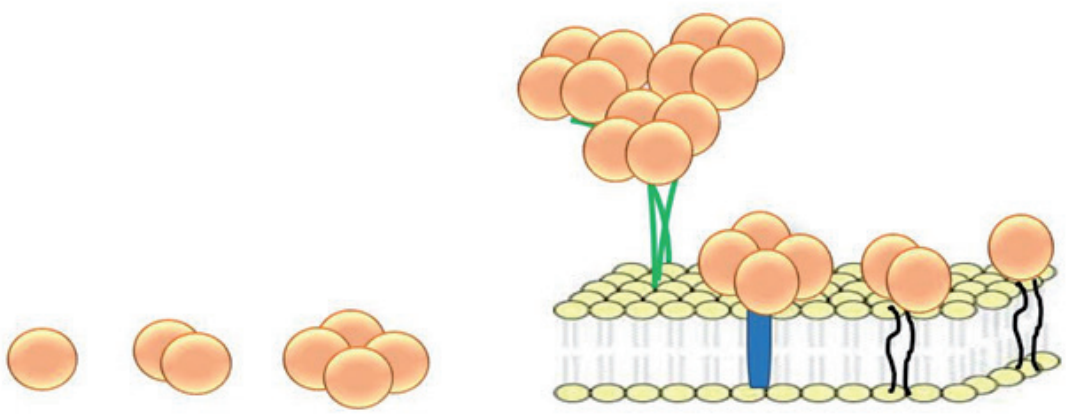

Fig. 2. Estructura del gen $B C H E$ humano, procesamiento de corte-empalme alternativo y productos proteicos. A) Los exones se muestran como rectángulos, los intrones como líneas horizontales. Región no codificante en los exones (color azul). B) Transcrito BuChE-T constituido por exones 2, 3, 4. C) Polimorfismo molecular de la proteína BuChE. Formas monoméricas; díméricas y tetraméricas globulares (subunidad catalítica en color gris). Formas asimétricas BuChE ancladas a membrana. La BuChE-T asociada a ColQ (líneas de color verde) o PRiMA (color azul). Monómeros y dímeros anclados a membrana a través de una alfa hélice (líneas de color negro) 
de mamíferos, la enzima predominante es la AChE, que realiza la hidrólisis de la acetilcolina. Además, en este órgano ambas colinesterasas se distribuyen de manera diferencial (Darvesh et al. 2003). Cuando se induce la inhibición de la actividad BuChE, ocurre un aumento en los niveles de la acetilcolina, evidenciando su papel regulador. Probablemente realiza funciones de filtro, esto es, colabora en la eliminación de un exceso de acetilcolina en las uniones nerviosas, evitando efectos deletéreos.

En una situación de carencia de actividad AChE, la proteína $\mathrm{BuChE}$ puede compensar algunas de las funciones en las que está implicada la primera, sobre todo aquellas relacionadas con el sistema colinérgico. Una evidencia que sustenta tal observación procede del desarrollo de animales transgénicos AChE-nulos en los cuales la actividad BuChE hidroliza a la acetilcolina (Li et al. 2000, Mesulam et al. 2002).

\section{Desintoxicación de venenos y drogas}

Se ha reportado que en algunos humanos completamente sanos no se presenta actividad BuChE en ningún tejido y/o órgano (Liddell et al. 1962, Doenicke et al. 1963). Esto condujo a suponer que la actividad de BuChE no cumplía un papel fisiológico.

Además, se desconoce el substrato endógeno de la BuChE (Çokuğraş, 2003). No obstante, la enzima puede hidrolizar compuestos que contienen esteres carboxílicos hidrofílicos o hidrofóbicos o ésteres de ácido fosfórico, por lo que se ha aceptado que la BuChE tiene un papel en la desintoxicación de venenos ingeridos o inhalados. Por ejemplo, la fisostigmina, incluida dentro de los inhibidores carbámicos, presente en las semillas de Physostigma venenosum conocida como haba de Calabar, y la cocaína presente en las hojas de la planta de coca, son tóxicos hidrolizados por la $\mathrm{BuChE}$ (Mattes et al. 1996). Los ésteres organofosforados (incluyendo los gases nerviosos, plaguicidas y anticolinesterásicos neurotóxicos denominados anatoxin-a(s) producidos por el alga azul-verdosa Anabaena flos-aquae) también son destruidos por la BuChE (Mahmood y Carmichael 1986).

La aspirina es hidrolizada por la BuChE para producir ácido salicílico (Masson et al. 1998). La heroína es un diéster que es convertido a morfina por la acción catalítica de la BuChE.

Broomfield et al. (1991) encontraron que monos pre-tratados con BuChE eran protegidos de la toxicidad de gases nerviosos (soman), lo cual indica que las propiedades de esta enzima la convierten en una "limpiadora biológica" que protege contra la toxicidad farmacológica y conductual de venenos organofosforados. Esta función desintoxicante confiere a la $\mathrm{BuChE}$ un papel relevante desde el punto de vista militar. Con base en resultados de experimentos en los que se ha administrado $\mathrm{BuChE}$ a distintas dosis a diversos tipos de animales, se ha establecido que éstos toleran de manera segura dosis que exceden los niveles endógenos en miles de veces (Saxena et al. 2011, Murthy et al. 2014).

Se considera, que la BuChE, a través de su actividad catalítica para hidrolizar distintos compuestos con capacidad anticolinesterásica, evita que éstos consigan llegar a la AChE localizada en sitios fisiológicos importantes (Çokuğraş 2003).

\section{LAS COLINESTERASAS, BLANCO DE LOS PLAGUICIDAS Y EFECTOS FISIOLÓGICOS RESULTANTES}

Los ésteres de organofosforados o carbamatos se usan como plaguicidas, agentes químicos para la guerra y drogas para el tratamiento del glaucoma, infecciones parasitarias y enfermedad de Alzheimer. Estos compuestos son inhibidores potentes de ambas colinesterasas. Los principales gases nerviosos tóxicos son soman, sarin y tabun, los cuales se utilizan usan contra poblaciones civiles y militares (Ganesan et al. 2010).

Según la Organización Mundial de la Salud, en países en vías de desarrollo anualmente mueren 200000 personas por envenenamiento con plaguicidas organofosforados (Eddleston et al. 2008). La inhibición progresiva de las colinesterasas por compuestos organofosforados (XPO[OR']) se debe a la fosforilación del residuo de serina en el sitio activo (BuChE Ser198 y AChE Ser200, para humanos). El organofosforado puede ser desplazado del sitio activo por nucleófilos fuertes (i.e., una oxima, representada como B), dejando libre a la enzima (Schwarz et al. 1995).

$\mathrm{XPO}\left(\mathrm{OR}^{\prime}\right)_{2}+\mathrm{EnzOH} \rightarrow \mathrm{EnzOPO}\left(\mathrm{OR}^{\prime}\right)_{2}+\mathrm{HX}$
$\mathrm{EnzOPO}\left(\mathrm{OR}^{\prime}\right) 2+\mathrm{B}: \rightarrow \mathrm{B}-\mathrm{PO}\left(\mathrm{OR}^{\prime}\right) \mathrm{z}+\mathrm{EnzOH}$

En la evaluación de exposiciones ocupacionales o ambientales a compuestos anti-colinesterásicos se estima la actividad AChE eritrocitaria y la actividad $\mathrm{BuChE}$ en suero o plasma. El nivel de inhibición de sus actividades varía entre los diferentes plaguicidas organofosforados. A este respecto, algunos poseen una mayor capacidad de inhibir la actividad $\mathrm{BuChE}$ que la actividad AChE. La inhibición de la primera se ha correlacionado con la intensidad y duración 
de exposiciones agudas a varios plaguicidas organofosforados y carbamatos (Araoud et al. 2011). Por otro lado, la inhibición de la actividad AChE es más sensible que la inhibición de BuChE en la exposición crónica a organofosforados (Kamel y Hoppin 2004).

Diversos estudios en poblaciones de trabajadores expuestos a compuestos organosfosforados, han informado que existe una relación entre la exposición a éstos y la inhibición de las actividades acetilcolinesterasa y butirilcolinesterasa, lo que provoca un incremento en la concentración de acetilcolina en los sitios de transmisión colinérgica, intensificando la acción de este neurotransmisor. Se ha establecido una relación entre la inhibición de la actividad AChE y los efectos en la salud. Una inhibición de 50 a $60 \%$ se asocia con un patrón dosis-respuesta a síntomas ligeros de debilidad, dolor de cabeza, náusea y salivación, con convalecencia de tres días. Por su parte, una inhibición de 60 a $90 \%$ produce síntomas como sudoración, vómito, diarrea, temblores, alteración de la marcha, dolor en el pecho y cianosis de las membranas mucosas que se revierten en pocas semanas. Una inhibición de 90 a $100 \%$ provoca la muerte por falla cardiaca o respiratoria (Maroni et al. 2000).

Respecto a lo anterior, se puede señalar que los efectos fisiológicos derivados de la exposición a compuestos anticolinesterásicos se relacionan con la capacidad de diversos tejidos u órganos para responder al neurotransmisor acetilcolina con el fin de desarrollar funciones no colinérgicas. Todos los tejidos no neuronales poseen la capacidad de capturar colina a través de un transportador que la ingresa al interior celular, para sintetizar la acetilcolina por acción de la colina acetiltransferasa, almacenar la acetilcolina en vesículas de secreción, liberarla hacia el medio extracelular, y responder a ella vía receptores muscarínicos y/o nicotínicos. Por ello se ha referido que poseen un sistema colinérgico no neural que los hace igualmente blanco de los efectos de inhibición por compuestos anticolinesterásicos.

\section{CONCLUSIÓN}

La inhibición de las actividades AChE y BuChE por compuestos organofosforados puede causar daños graves permanentes sobre la salud humana, debido a que la disminución en sus actividades enzimáticas provocaría aumentos considerables en las concentraciones de acetilcolina, lo cual causaría la aparición del síndrome colinérgico. El cuadro de intoxicación por organofosforados genera un conjunto de síntomas tales como salivación, lagrimeo, diarrea, calambres abdominales, náusea y vómito (efectos muscarínicos), fasciculación de los músculos y calambres, debilidad muscular, taquicardia, hipertensión (efectos nicotínicos), y agitación, confusión, coma y depresión respiratoria (efectos del sistema nervioso central). En cuanto a la actividad de la acetilcolina como factor de proliferación podría tener una fuerte participación en la génesis del cáncer, no porque sea un carcinogénico sino por su capacidad mitogénica, lo cual la convierte en el agente proliferativo que necesita una célula mutada para proliferar y que se establezca la mutación requerida para la aparición y el desarrollo de un tumor.

\section{AGRADECIMIENTOS}

Los autores agradecen el financiamiento recibido por el Programa para el Desarrollo Profesional Docente, para el Tipo Superior (PRODEP), de la Dirección de Superación Académica de la Secretaria de Educación Pública a través del Programa de Fortalecimiento de Cuerpos Académicos convenio núm. DSA/1035/16/9856. Asimismo, a la Red Temática de Toxicología de Plaguicidas (CONACyT-262284/280045/294303).

\section{REFERENCIAS}

Alavanja M.C., Hoppin J.A. y Kamel F. (2004). Health effects of chronic pesticide exposure: cancer and neurotoxicity. Annu. Rev. Public Health 25, 155-197. DOI: 10.1146/annurev.publhealth.25.101802.123020

Allderdice P.W., Gardner H.A., Galutira D., Lockridge O., LaDu B.N. y McAlpine P.J. (1991). The cloned butyrylcholinesterase (BCHE) gene maps to a single chromosome site, 3q26. Genomics 11, 452-454.

DOI: 10.1016/0888-7543(91)90154-7

Alles G.A. y Hawes R.C. (1940). Cholinesterases in the blood of man. J. Biol. Chem. 133, 375-390.

Araoud M., Neffeti F., Douki W., Hfaiedh H.B., Akrout M., Najjar M.F. y Kenani A. (2011). Factors influencing plasma butyrylcholinesterase activity in agricultural workers. Ann. Biol. Clin. 69, 159-166.

DOI: $10.1684 / \mathrm{abc} .2011 .0531$

Ariel N., Ordentlich A., Barak D., Bino T., Velan B. y Shafferman A. (1998). The 'aromatic patch' of three proximal residues in the human acetylcholinesterase active centre allows for versatile interaction modes with inhibitors. Biochem. J. 335, 95-102.

DOI: $10.1042 / \mathrm{bj} 3350095$ 
Arpagaus M., Kott M., Vatsis K.P., Bartels C.F., La Du B.N. y Lockridge O. (1990). Structure of the gene for human butyrylcholinesterase. Evidence for a single copy. Biochemistry 29, 124-131.

Bernardi C.C., Ribeiro E. de S., Cavalli I.J., ChautardFreire-Maia E.A. y Souza R.L. (2010). Amplification and deletion of the ACHE and BCHE cholinesterase genes in sporadic breast cancer. Cancer Genet. Cytogenet. 197, 158-165.

DOI: 10.1016/j.cancergencyto.2009.10.011.

Bon S., Coussen F. y Massoulié J. (1997). Quaternary associations of acetylcholinesterase. II. The polyproline attachment domain of the collagen tail. J. Biol. Chem. 272, 3016-3021.

DOI: $10.1074 / \mathrm{jbc} .272 .5 .3016$

Broomfield C.A., Maxwell D.M., Solana R.P., Castro C.A., Finger A.V. y Lenz D.E (1991). Protection by butyrylcholinesterase against organophosphorus poisoning in nonhuman primates. J. Pharmacol. Exp. Ther. 259, 633-638.

Cassiman D., Libbrecht L., Sinelli N., Desmet V., Denef C. y Roskams T. (2002). The vagal nerve stimulates activation of the hepatic progenitor cell compartment via muscarinic acetylcholine receptor type 3. Am. J. Pathol. 161, 521-530.

DOI: $10.1016 / \mathrm{S} 0002-9440(10) 64208-3$

Cohen J.A. y Oosterbaan R.A. (1963). The active site of acetylcholinesterase and related esterases and its reactivity toward substrates and inhibitors En: Cholinesterases and anticholinesterase agents (Koelle G.K., Ed.). Springer-Verlag. Berlín. pp. 299-373.

Çokuğraş N.A. (2003). Butyrylcholinesterase: Structure and physiological importance. Turk. J. Biochem. 28, 54-61.

Coussen F., Ayon A., Le Goff A., Leroy J., Massoulie J. y Bon S. (2001). Addition of a glycophosphatidylinositol to acetylcholinesterase. Processing, degradation, and secretion. J. Biol. Chem. 276, 27881-27892.

DOI: $10.1074 /$ jbc.M010817200

Dale H.H. (1914). The action of certain esters and ethers of choline, and their relation to muscarine. J. Pharmacol. Exp. Therap. 6, 147-190.

Darvesh S., Hopkins D.A. y Geula, C. (2003). Neurobiology of butyrylcholinesterase. Nat. Rev. Neurosci. 4, 131-138. DOI: 10.1038/nrn1035

Doenicke A., Gurtner T., Kreutzberg G., Remes I. y Spiess W. y Steinbereithner K. (1963). Serum cholinesterase anenzymia. Report of a case confirmed by enzymehistochemical examination of liver-biopsy specimen. Acta Anaesthesiol. Scand. 7, 59-68. DOI: $10.1111 / j .1399-6576.1963 . t b 00204 . x$

Dvir H., Silman I., Harel M., Rosenberry T.L. y Sussman J.L. (2010). Acetylcholinesterase: from 3D structure to function. Chem. Biol. Interact. 187, 10-22.

DOI: 10.1016/j.cbi.2010.01.042

Eddleston M., Buckley N.A., Eyer P. y Dawson AH. (2008). Management of acute organophosphorus pesticide poisoning. Lancet 371, 597-607.

DOI: 10.1016/S0140-6736(07)61202-1

Fidder A., Hulst A.G., Noort D., de Ruiter R., van der Schans M.J., Benschop H.P. y Langenberg J.P. (2002). Retrospective detection of exposure to organophosphorus anti-cholinesterases: mass spectrometric analysis of phosphylated human butyrylcholinesterase. Chem. Res. Toxicol. 15, 582-590. DOI: 10.1021/tx0101806

Gahler O. y Plattner F. (1927). I. Fate of acetylcholine in the blood. Arch. Ges. Physiol. 218, 488-505.

Ganesan K., Raza S.K. y Vijayaraghavan R. (2010). Chemical warfare agents. J. Pharm. Bioallied Sci. 2, 166-178. DOI: 10.4103/0975-7406.68498

Gaughan G., Park H., Priddle J., Craig I. y Craig S. (1991). Refinement of the localization of human butyrylcholinesterase to chromosome 3q26.1-q26.2 using a PCRderived probe. Genomics 11, 455-458. DOI: $10.1016 / 0888-7543(91) 90155-8$

Genever P.G., Birch M.A., Brown E. y Skerry T.M. (1999). Osteoblast-derived acetylcholinesterase: a novel mediator of cell-matrix interactions in bone? Bone 24, 297-303. DOI: 10.1016/S8756-3282(98)00187-2

Getman D.K., Eubanks J.H., Camp S., Evans G.A. y Taylor P. (1992). The human gene encoding acetylcholinesterase is located on the long arm of chromosome 7. Am. J. Hum. Genet. 51, 170-177.

Glick D. (1941). Some additional observations on the specificity of cholinesterase. J. Biol. Chem. 137, 456-460.

Gómez J.L., Moral-Naranjo M.T., Campoy F.J. y Vidal C.J. (1999). Characterization of acetylcholinesterase and butyrylcholinesterase forms in normal and dystrophic Lama2dy mouse heart. J. Neurosci. Res. 56, 295-306. DOI: 10.1002/(SICI)1097-4547(19990501)56:3<295: AID-JNR9>3.0.CO;2-J

Gómez J.L., García-Ayllón M.S., Campor F.J. y Vidal C.J. (2000). Muscular dystrophy alters the processing of light acetylcholinesterase but not butyrylcholinesterase forms in liver of Lama2dy mice. J. Neurosci. Res. 62, 134-145. DOI: 10.1002/10974547(20001001)62:1<134: AID-JNR14>3.0.CO;2-T

Gómez J.L., Nieto-Cerón S., Campoy F.J., Muñoz-Delgado E. y Vidal C.J. (2003). Purification and properties of hydrophilic dimers of acetylcholinesterase from mouse erythrocytes. Int. J. Biochem. Cell Biol. 35, 1109-1118. DOI: $10.1016 / \mathrm{S} 1357-2725(03) 00007-4$

Jansz H.S., Brons D. y Warringa M.G.P.J. (1959). Chemical nature of the DFP-binding site of pseudocholinesterase Biochim. Biophys. Acta 34, 573-575.

DOI: 10.1016/0006-3002(59)90320-8 
Jin Q.H., He H.Y., Shi Y.F., Lu H. y Zhang X.J. (2004). Overexpression of acetylcholinesterase inhibited cell proliferation and promoted apoptosis in NRK cells. Acta Pharmacol. Sinica 25, 1013-1021.

Kalow W. y Staron N. (1957). On distribution and inheritance of atypical forms of human serum cholinesterase, as indicated by dibucaine numbers. Can. J. Med. Sci. 35, 1305-1320.

Kamel F. y Hoppin J.A. (2004). Association of pesticide exposure with neurologic dysfunction and disease. Environ. Health Perspect. 112, 950-958. DOI: $10.1289 /$ ehp.7135

Kaufer D., Friedman A., Seidman S. y Soreq H. (1998). Acute stress facilitates long-lasting changes in cholinergic gene expression. Nature 393, 373-337.

DOI: $10.1038 / 30741$

La Du B.N., Bartels C.F., Nogueira C.P., Hajra A., Lightstone H., Van der Spek A. y Lockridge O. (1990). Phenotypic and molecular biological analysis of human butyrylcholinesterase variants. Clin. Biochem. 23, 423-431. DOI: 10.1016/0009-9120(90)90187-Y

Layer, P. G. y Willbold, E. (1995). Novel functions of cholinesterases in development, physiology and disease. Prog. Histochem. Cytochem. 29, 1-94.

Layer P.G., Klaczinski J., Salfelder A., Sperling L.E., Thangaraj G., Tuschl C. y Vogel-Höpker A. (2013). Cholinesterases in development: $\mathrm{AChE}$ as a firewall to inhibit cell proliferation and support differentiation. Chem. Biol. Interact. 203, 269-276.

DOI: 10.1016/j.cbi.2012.09.014

Lehmann H. y Ryan E. (1956). The familial incidence of low pseudocholinesterase level. Lancet 271, 124. DOI: 10.1016/S0140-6736(56)90869-8

Lev-Lehman E., Deutsch V., Eldor A. y Soreq H. (1997). Immature human megakaryocytes produce nuclear-associated acetylcholinesterase. Blood 89, 3644-3653.

Li B., Stribley J.A., Ticu A., Xie M., Schopfer L.M., Hammond P., Brimijoin S., Hinrichs S.H. y Lockridge O. (2000). Abundant tissue butyrylcholinesterase and its possible function in the acetylcholinesterase knockout mouse. J. Neurochem. 75, 1320-1331. DOI: $10.1046 /$ j.1471-4159.2000.751320.x

Liddell J., Lehmann H. y Silk E. (1962). A 'silent' pseudo-cholinesterase gene. Nature 193, 561-562. DOI: 10.1038/193561a0

Lockridge O. (2015). Review of human butyrylcholinesterase structure, function, genetic variants, history of use in the clinic, and potential therapeutic uses. Pharmacol. Terap. 148, 34-48. DOI: $10.1016 /$ j.pharmthera.2014.11.011

Mahmood N.A. y Carmichael W.W. (1986). The pharmacology of anatoxin-a(s), a neurotoxin produced by the freshwater cyanobacterium Anabaena flos-aquae NRC 525-517. Toxicon. 24, 425-434.

DOI: 10.1016/0041-0101(86)90074-7

Maroni M., Colosio C., Ferioli A. y Fait A. (2000). Biological monitoring of pesticide exposure: A review. Introduction. Toxicology 143, 1-118. DOI: 10.1016/S0300-483X(99)00152-3

Masson P., Froment M.T., Fortier P.L., Visicchio J.E., Bartels C.F. y Lockridge O. (1998). Butyrylcholinesterasecatalysed hydrolysis of aspirin, a negatively charged ester, and aspirin-related neutral esters. Biochim. Biophys. Acta 1387, 41-52.

DOI: 10.1016/S0167-4838(98)00104-6

Massoulié J. y Toutant J.P. (1988) Vertebrate cholinesterases: Structure and types of interaction. En: The cholinergic synapse. Handbook of experimental pharmacology (Whittaker V.P., Ed.). Springer, Berlin, Heidelberg, pp 167-224.

DOI: 10.1007/978-3-642-73220-1_8

Massoulié J., Pezzementi L., Bon S., Krejci E. y Vallette, F.M. (1993). Molecular and cellular biology of cholinesterases. Prog. Neurobiol. 41, 31-91.

DOI: 10.1016/0301-0082(93)90040-Y

Massoulié J., Anselmet A., Bon S., Krejci E., Legay C., Morel N. y Simon S. (1998). Acetylcholinesterase: C-terminal domains, molecular forms and functional localization. J. Physiol. 92, 183-190.

DOI: $10.1016 / \mathrm{S} 0928-4257(98) 80007-7$

Massoulie J. (2002). The origin of the molecular diversity and functional anchoring of cholinesterases. Neurosignals 11, 130-43. DOI:10.1159/000065054

Mattes C., Bradley R., Slaughter E. y Browne S. (1996). Cocaine and butyrylcholinesterase (BChE): Determination of enzymatic parameters. Life Sci. 58, PL257PL261. DOI: 10.1016/0024-3205(96)00065-3

McGuire M.C., Nogueira C.P., Bartels C.F., Lightstone H., Hajra A., van der Spek A.F., Lockridge O. y La Du B.N. (1989). Identification of the structural mutation responsible for the dibucaine-resistant (atypical) variant form of human serum cholinesterase. Proc. Natl. Acad. Sci. USA 86, 953-957.

DOI: $10.1073 /$ pnas.86.3.953

Meshorer E., Erb C., Gazit R., Pavlovsky L., Kaufer D., Friedman A. Glick D., Ben-Arie N. y Soreq H. (2002). Alternative splicing and neuritic mRNA translocation under long-term neuronal hypersensitivity. Science 295, 508-512. DOI: 10.1126/science. 1066752

Meshorer E., Toiber D., Zurel D., Sahly I., Dori A., Cagnano E. Schreiber L., Grisaru D., Tronche F. y Soreq H. (2004). Combinatorial complexity of 5' alternative acetylcholinesterase transcripts and protein products. J. Biol. Chem. 279, 29740-29751. DOI: $10.1074 /$ jbc.M402752200 
Mesulam M.-M. Guillozet A., Shaw P., Levey A., Duysen E.G. y Lockridge O. (2002). Acetylcholinesterase knockouts establish central cholinergic pathways and can use butyrylcholinesterase to hydrolyze acetylcholine. Neurosci. 110, 627-639 DOI: 10.1016/S03064522(01)00613-3

Montenegro M.F., Nieto-Cerón S., Cabezas-Herrera J., Muñoz-Delgado E., Campoy F.J. y Vidal C.J. (2014). Most acetylcholinesterase activity of non-nervous tissues and cells arise from the AChE-H transcripts. J. Mol. Neurosci. 53, 429-435.

DOI: $10.1007 / \mathrm{s} 12031-013-0172-8$

Moral-Naranjo M.T., Montenegro M.F., Muñoz-Delgado E., Campoy F.J. y Vidal C.J. (2010). The levels of both lipid rafts and acetylcholinesterase raft-located acetylcholinesterase dimers increase in muscle of mice with muscular dystrophy by merosin deficiency. Biochim. Biophys. Acta. 1802, 754-764.

DOI: 10.1016/j.bbadis. 2010.05.011

Muñoz-Delgado E., Montenegro M.F., Campoy F.J., Moral-Naranjo M.T., Cabezas-Herrera J. y Kovacs G. (2010). Expression of cholinesterases in human kidney and its variation in renal cell carcinoma types. FEBS J. 277, 4519-4529. DOI:10.1111/j.1742-4658.2010.07861.x

Murthy V., Gao Y., Geng L., LeBrasseur N.K., White T.A., Parks R.J. y Brimijoin S. (2014). Physiologic and metabolic safety of butyrylcholinesterase gene therapy in mice. Vaccine 32, 4155-4162.

DOI: $10.1016 /$ j.vaccine.2014.05.067

Nachmansohn D. y Wilson I.B. (1951). The enzymic hydrolysis and synthesis of acetylcholine. Adv. Enzymol. Relat. Subj. Biochem. 12, 259-339.

Navaranjan G., Hohenadel K., Blair A., Demers P.A., Spinelli J.J., Pahwa P., McLaughlin J.R., Dosman J.A., Ritter L. y Harris S.A. (2013). Exposures to multiple pesticides and the risk of Hodgkin lymphoma in Canadian men. Cancer Cause. Control 24, 1661-1673. DOI: $10.1007 / \mathrm{s} 10552-013-0240-\mathrm{y}$

Nicolet Y., Lockridge O., Masson P., Fontecilla-Camps J.C. y Nachon F. (2003). Crystal structure of human butyrylcholinesterase and of its complexes with substrate and products. J. Biol. Chem. 278, 41141-41147. DOI: 10.1074/jbc.M210241200

Nieto-Cerón S., del Campo L.F., Muñoz-Delgado E., Vidal C.J. y Campoy F.J. (2005). Muscular dystrophy by merosin deficiency decreases acetylcholinesterase activity in thymus of Lama2dy mice. J. Neurochem. 95, 1035-1046. DOI: 10.1111/j.1471-4159.2005.03433.x

Ordentlich A., Barak D., Kronman C., Flashner Y., Leitner M., Segall Y., Ariel N., Cohen S., Velan B. y Shafferman A. (1993). Dissection of the human acetylcholinesterase active center determinants of substrate specificity. Identification of residues constituting the anionic site, the hydrophobic site, and the acyl pocket. J. Biol. Chem. 268, 17083-17095.

Ordentlich A, Barak D., Kronman C., Ariel N., Segall Y., Velan B. y Shafferman A. (1998). Functional characteristics of the oxyanion hole in human acetylcholinesterase. J. Biol. Chem. 273, 19509-19517.

DOI: $10.1074 / j b c .273 .31 .19509$

Park S.E., Kim N.D. y Yoo Y.H. (2004). Acetylcholinesterase plays a pivotal role in apoptosome formation. Cancer Res. 64, 2652-2655. DOI: 10.1158/0008-5472.CAN-04-0649

Park S.E., Jeong S.H., Yee S.B., Kim T.H., Soung Y.H., Ha N.C., Kim N.D., Park J.Y., Bae H.R., Park S.S., Lee H.J. y Yoo Y.H. (2008). Interactions of acetylcholinesterase with caveolin-1 and subsequently with cytochrome $\mathrm{c}$ are required for apoptosome formation. Carcinogenesis 29, 729-737.

DOI: $10.1093 /$ carcin/bgn036

Pérez-Aguilar B., Vidal C.J., Palomec G., García-Dolores F., Gutiérrez-Ruiz M.C., Bucio L., Gómez-Olivares J.L. y Gómez-Quiroz L.E. (2015). Acetylcholinesterase is associated with a decrease in cell proliferation of hepatocellular carcinoma cells. Biochim. Biophys. Acta. 1852, 1380-1387.

DOI: 10.1016/j.bbadis.2015.04.003

Perrier A.L., Massoulie J. y Krejci E. (2002). PRiMA: the membrane anchor of acetylcholinesterase in the brain. Neuron 33, 275-85. DOI: $10.1016 / \mathrm{S} 0896-6273(01) 00584-0$

Pohanka M. (2011). Cholinesterases, a target of pharmacology and toxicology. Biomed. Pap. Med. Fac. Univ. Palacky Olomouc Czech Repub. 155, 219-229.

DOI: $10.5507 /$ bp.2011.036

Quinn D.M. (1987). Acetylcholinesterase: enzyme structure, reaction dynamics, and virtual transition states. Chemical Rev. 87, 955-979.

DOI: $10.1021 / \mathrm{cr} 00081 \mathrm{a} 005$.

Radic Z., Gibney G., Kawamoto S., MacPhee-Quigley K., Bongiorno C. y Taylor P. (1992). Expression of recombinant acetylcholinesterase in a baculovirus system: kinetic properties of glutamate 199 mutants. Biochemistry 31, 9760-9767. DOI: 10.1021/bi00155a032

Radic Z., Pickering N.A., Vellom D.C., Camp S. y Taylor P. (1993). Three distinct domains in the cholinesterase molecule confer selectivity for acetyl- and butyrylcholinesterase inhibitors. Biochemistry 32, 12074-12084. DOI: 10.1021/bi00096a018

Rosenberry T.L. (1979). Quantitative simulation of endplate currents at neuromuscular junctions based on the reaction of acetylcholine with acetylcholine receptor and acetylcholinesterase. Biophys J. 26, 263-289. DOI: $10.1016 / \mathrm{S} 0006-3495(79) 85249-2$ 
Ruiz-Espejo F., Cabezas-Herrera J., Illana J., Campoy F. J. y Vidal C.J. (2002). Cholinesterase activity and acetylcholinesterase glycosylation are altered in human breast cancer. Breast Cancer Res. Treat. 72, 11-22. DOI: 10.1023/A: 1014904701723

Saxena A., Sun W., Fedorko J.M., Koplovitz I. y Doctor B.P. (2011). Prophylaxis with human serum butyrylcholinesterase protects guinea pigs exposed to multiple lethal doses of soman or VX. Biochem. Pharmacol. 81, 164-169. DOI: 10.1016/j.bcp.2010.09.007.

Schwarz M., Glick D., Loewenstein Y. y Soreq H. (1995). Engineering of human cholinesterases explains and predicts diverse consequences of administration of various drugs and poisons. Pharmac. Ther. 67, 283-322.

Serobyan N., Jagannathan S., Orlovskaya I., Schraufstatter I., Skok M., Loring J. y Khaldoyanidi S. (2007). The cholinergic system is involved in regulation of the development of the hematopoietic system. Life Sci. 80, 2352-2560. DOI: 10.1016/j.lfs.2007.04.017

Soreq F., Lapidot-Lifson Y. y Zakut H. (1991). A role for cholinesterases in tumorigenesis? Cancer Cells 3, 511-516.

Soreq H. y Seidman S. (2001). Acetylcholinesterase-new roles for an old actor. Nat. Rev. Neurosci. 2, 294-302. DOI: $10.1038 / 35067589$

Stedman E., Stedman E. y Eason L., H. (1932). Choline esterase. An enzyme present in the blood serum of the horse. Biochem. J. 26, 2056-2066.

Sussman J.L., Harel M., Frolow F., Oefner C., Goldman A., Toker L. y Silman I. (1991). Atomic structure of acetylcholinesterase from Torpedo californica: a prototypic acetylcholine-binding protein. Science 253, 872-879. DOI: 10.1126/science. 1678899

Sussman J.L., Harel M. y Silman I. (1993). Three-dimensional structure of acetylcholinesterase and of its complexes with anticholinesterase drugs. Chem. Biol. Interact. 87, 187-197.

DOI: 10.1016/0009-2797(93)90042-W
Taylor P. y Lappi S. (1975). Interaction of fluorescence probes with acetylcholinesterase. The site and specificity of propidium binding. Biochemistry 14, 1989-1997. DOI: 10.1021/bi00680a029

Taylor P. (1991). The cholinesterases. J. Biol. Chem. 266, 4025-4028.

Taylor P. y Radic Z. (1994). The cholinesterases: from genes to proteins. Annu. Rev. Pharmacol. Toxicol. 34, 281-320. DOI: 10.1146/annurev.pa.34.040194. 001433

Thunnissen F.B. (2009). Acetylcholine receptor pathway and lung cancer. J. Thorac. Oncol. 4, 943-946. DOI: $10.1097 /$ JTO.0b013e3181ad83fc

Triggle D.J., Mitchell J.M. y Filler R. (1998). The pharmacology of physostigmine. CNS Drug Rev. 4, 87-136. DOI: 10.1111/j.1527-3458.1998.tb00059.x

Toiber D., Greenberg D.S. y Soreq H. (2009). Proapoptotic protein-protein interactions of the extended N-AChE terminus. J. Neural Transm. 116, 1435-1442. DOI: $10.1007 / \mathrm{s} 00702-009-0249-2$

Vidal C.J. (2005). Expression of cholinesterases in brain and non-brain tumours. Chem. Biol. Interact. 157-158, 227-232. DOI: 10.1016/j.cbi.2005.10.035

Xiang A.C., Xie J. y Zhang X.J. (2008). Acetylcholinesterase in intestinal cell differentiation involves G2/M cell cycle arrest. Cel. Mol. Life Sci. 65, 1768-1779. DOI: $10.1007 / \mathrm{s} 00018-008-8016-3$

Yang L., He H.Y. y Zhang X.J. (2002). Increased expression of intranuclear AChE involved in apoptosis of SK-N-SH cells. Neurosci. Res. 42, 261-268. DOI: 10.1016/ s0168-0102(02)00005-6

Zhang X.J., Yang L., Zhao Q., Caen J.P., He H.Y. Jin QH, Guo L.H., Alemany M., Zhang L.Y. y Shi Y.F. (2002). Induction of acetylcholinesterase expression during apoptosis in various cell types. Cell Death Differ. 9, 790-800. DOI: 10.1038/sj.cdd.4401034 